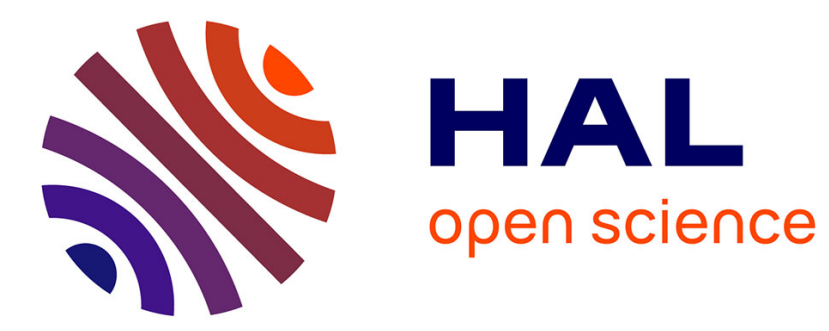

\title{
Channel Estimation Impact for LTE Small Cells based on MU-VFDM
}

Marco Maso, Leonardo S. Cardoso, Mérouane Debbah, Lorenzo Vangelista

\section{To cite this version:}

Marco Maso, Leonardo S. Cardoso, Mérouane Debbah, Lorenzo Vangelista. Channel Estimation Impact for LTE Small Cells based on MU-VFDM. IEEE WCNC 2012, Apr 2012, Paris, France. pp.2560 - 2565, 10.1109/WCNC.2012.6214230 . hal-00769463

\section{HAL Id: hal-00769463 \\ https://hal-centralesupelec.archives-ouvertes.fr/hal-00769463}

Submitted on 8 Jan 2013

HAL is a multi-disciplinary open access archive for the deposit and dissemination of scientific research documents, whether they are published or not. The documents may come from teaching and research institutions in France or abroad, or from public or private research centers.
L'archive ouverte pluridisciplinaire HAL, est destinée au dépôt et à la diffusion de documents scientifiques de niveau recherche, publiés ou non, émanant des établissements d'enseignement et de recherche français ou étrangers, des laboratoires publics ou privés. 


\title{
Channel Estimation Impact for LTE Small Cells based on MU-VFDM
}

\author{
Marco Maso $^{\star, \diamond}$, Leonardo S. Cardoso ${ }^{\dagger}$, Mérouane Debbah $^{\star}$ and Lorenzo Vangelista \\ $\star$ Alcatel-Lucent Chair - SUPÉLEC, Gif-sur-Yvette, France \\ $\diamond$ Department of Information Engineering, University of Padova, Italy \\ $\dagger$ INSA - Lyon, France \\ \{marco.maso, merouane.debbah\}@supelec.fr, leonardo.sampaio-cardoso@inria.fr, lorenzo.vangelista@unipd.it
}

\begin{abstract}
In a previous work, we introduced a spectrum sharing technique called Multi-User Vandermonde-subspace Frequency Division Multiplexing (MU-VFDM). This overlay technique allows the coexistence of a downlink Orthogonal Frequency Division Multiple Access (OFDMA) macro-cell and a cognitive multi-user small-cell system in time division duplex mode. In that work, MU-VFDM was shown to be able to completely cancel the interference towards a macro-cell system at the price of perfect channel state information (CSI) at the opportunistic small-cells. In this work we relax the perfect CSI constraint by introducing a channel estimation protocol that does not require cooperation between the two systems, but still provides harmless coexistence between them. The impact of this protocol is evaluated in terms of interference at the legacy and sum-rates at the opportunistic system. Simulation results show that, even with imperfect CSI estimation, MU-VFDM is able to achieve promising rates for the small-cells while incurring a small rate loss at the macro-cell due to interference.
\end{abstract}

Index Terms-small-cells, channel estimation, VFDM, interference cancelation, overlay cognitive network

\section{INTRODUCTION}

With the aging of current $3 \mathrm{G}$ cellular networks, Long Term Evolution (LTE), is seen by 3GPP as 3G's natural evolution path [1]. In spite of its new features, LTE is still plagued by some of the same restrictions its predecessors faced. Some of these restrictions, such as limited coverage and capacity, come as a consequence of the cellular structure itself, inherited from older generation cellular systems. The common way to address these limitations is by using a heterogeneous network structure, in which another radio access technology (i.e., 802.11) is employed alongside the cellular one to address a poor coverage area or a high concentration of subscribers. Unfortunately, heterogeneous networks rely on seamless switching between radio protocol stacks (known as vertical handovers), which has failed to gain thorough commercial adoption.

Recently, the concept of Small Cells (SCs) has been introduced to describe the family of all the short-range radio cells such as femto/pico/micro-cells, using cognition and awareness to effectively break off from the cellular structure constraints, increasing outdoor and indoor capacities [2]. Deployed in a massively dense network of self-organizing and pervasive low powered radio nodes, SCs (such as the LightRadios [3]) are not limited to indoor use and can be installed on top of lamp-posts, trees and other existing urban structures. Clearly, deploying a dense second tier wireless network that shares the same band as the first tier, without cooperating with it, will produce unacceptable amounts of interference to the latter, if unmanaged. Solutions to allow SCs to coexist with Macro Cells (MCs) using the same frequency band, are still subject of research. One candidate solution is the Dynamic Spectrum Access (DSA) based on cognitive radio [4]. DSA can be implemented through various paradigms, namely overlay, underlay and interweave [4]. These paradigms are characterized mainly by the approach taken to the interference control [5], [6], [7].

In [8], [9] we proposed an overlay DSA technique, called MU-VFDM, to deal with inter-SC interference. MU-VFDM makes use of frequency selectivity and the redundancy introduced to combat inter block interference, to deal with the interference from the secondary (opportunistic) to the primary system (licensee). This is accomplished by creating a precoder that is orthogonal to the interfering link. The interference cancelation provided by MU-VFDM does not require cooperation between the two tiers and holds true for any transmit power, either at the MC or SCs, unlike the underlay-based approaches. We have further shown that we are able to guarantee a perfect interference cancelation under the assumption of perfect CSI. In this contribution, we consider the same two-tiered network composed of an LTE-MC system and an LTE-SC system transmitting in Time Division Duplex (TDD) mode. We relax the assumptions of perfect CSI, made in previous works, to design a suitable channel estimation procedure for MU-VFDM. Lastly, we discuss the impact of this new channel estimation procedure and study the best proportion of training symbols versus data symbols required to achieve the best performance.

This paper is organized as follows. In Sec. II, we introduce the general MC/SC model. We introduce the inter-SC interference management scheme in Sec. III. In Sec. IV, we present the channel estimation procedure. In Sec. V, we show some numerical results for our MC/SC study case based on LTE. Finally, conclusions and future research directions are discussed in Sec. VI.

\section{SYSTEM MODEL}

Consider the downlink model in Fig. 1 where, an LTE $\mathrm{MC}$ and $K$ SCs share the same frequency band, with no 
cooperation. The LTE MC is composed of a Macro-cell Base Station (MBS) and $M$ User Equipments (MUEs). We assume that Small-cell Access Points (SAPs) may communicate over an infinite backhaul capacity, realizing a fully coordinated network Multiple Input Multiple Output (MIMO). Nonetheless, we remark that the two tiers are totally independent and disconnected, and hence they are not parts of a MultiUser MIMO (MU-MIMO) system [10]. For simplicity, but without loss of generality, we consider that each SC hosts only one single antenna Small-cell User Equipment (SUE). We note that, a multi-SUE extension could be easily obtained adopting techniques as the ones described in [10]. Throughout

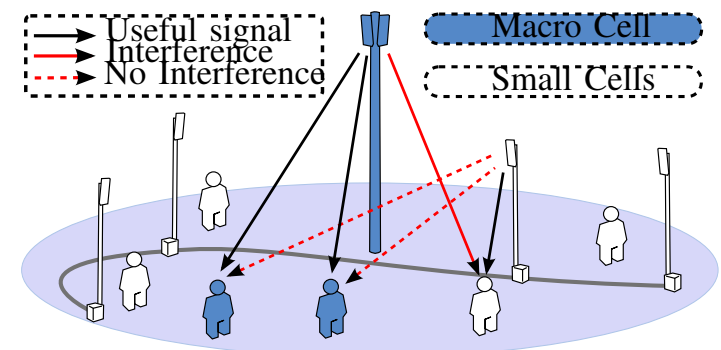

Figure 1. MU-VFDM downlink model, two-tiered network.

this work, subscript "m" refers to the MC, while "s" refers to the SCs, i.e., $h_{\mathrm{sm}}^{(i, j)}\left(\right.$ or $\mathbf{H}_{\mathrm{sm}}^{(i, j)}$ ) denotes a link from SAP $i$ to MUE $j . \mathbf{s}^{[i]}\left(\right.$ or $\mathbf{H}_{\mathrm{sm}}^{([i], j)}$ ) denotes a vector/matrix related to the transmission from any SAP except $i$. All channel vectors $\mathbf{h}_{\mathrm{ab}}^{(\cdot, \cdot)} \in \mathcal{C N}\left(0, \mathbf{I}_{L+1} /(L+1)\right)$ represent the impulse response of independent and identically distributed (i.i.d.) frequencyselective Rayleigh fading channels composed of $L+1$ paths.

Both systems adopt a block transmission scheme. An $M$ user OFDMA based transmission of block size $N+L$, of which $L$ is the length of the cyclic prefix, is adopted in the MC. As such, each MUE and SUE discards the leading $L$ symbols and performs a Discrete Fourier Transform (DFT). Conceptually, the only distinction between an MUE and a SUE, is the connection to the respective MBS/SAP. For the $\mathrm{MC}$, a uniform resource allocation is adopted, i.e., $N / M$ subcarriers per MUE. Let $[\cdot]_{m, n}$ denote a matrix element at the $m^{\text {th }}$ row and the $n^{\text {th }}$ column. We define $\mathbf{B}_{j}$ as the mask filter used by MUE $j$ to select its allocated subcarriers, s.t. $\left[\mathbf{B}_{j}\right]_{(n, n)}=1$ if the subcarrier $n$ is allocated to the MUE $j$ and zero otherwise.

Let $\otimes$ denote the Kronecker product, $\mathbf{I}_{N}$ the identity matrix of size $\mathrm{N}$, and $\mathbf{F} \in \mathcal{C}^{N \times N}$ a unitary DFT matrix with $[\mathbf{F}]_{(k+1, l+1)}=\frac{1}{\sqrt{N}} e^{-i 2 \pi \frac{k l}{N}}$ for $k, l=[0, N-1]$. We define $\mathbf{s}_{\mathrm{m}} \triangleq\left[\mathbf{s}_{\mathrm{m}}^{(1) \mathrm{T}}, \ldots, \mathbf{s}_{\mathrm{m}}^{(M) \mathrm{T}}\right]^{\mathrm{T}}$ as the overall MC transmit vector of size N. Similarly, we define $\mathbf{s}_{\mathrm{s}} \triangleq\left[\mathbf{s}_{\mathrm{s}}^{(1) \mathrm{T}}, \ldots, \mathbf{s}_{\mathrm{s}}^{(K) \mathrm{T}}\right]^{\mathrm{T}}$ as the overall SC transmit vector, whose size will be discussed later. Let $\mathbf{y}_{\mathrm{m}}^{(j)}, \mathbf{y}_{\mathrm{s}}^{(j)}$ be the received signal at the $\mathrm{j}^{\text {th }}$ MUE/SUE, of dimension $N$ and $K N$ respectively. As a consequence, $\mathbf{y}_{\mathrm{m}}=\sum_{j=1}^{M} \mathbf{y}_{\mathrm{m}}^{(j)}$ is the overall received vector at the MUEs of size $\mathrm{N}$ and $\mathbf{y}_{\mathrm{s}} \triangleq\left[\mathbf{y}_{\mathrm{s}}^{(1) \mathrm{T}}, \ldots, \mathbf{y}_{\mathrm{s}}^{(K) \mathrm{T}}\right]^{\mathrm{T}}$, the overall received vector at the SUEs obtained by aggregating each single user component. We can write

$$
\begin{aligned}
\mathbf{y}_{\mathrm{m}} & =\mathbf{H}_{\mathrm{mm}} \mathbf{s}_{\mathrm{m}}+\mathbf{H}_{\mathrm{sm}} \mathbf{E s}_{\mathrm{s}}+\mathbf{F n}_{\mathrm{m}} \\
\mathbf{y}_{\mathrm{s}} & =\mathbf{H}_{\mathrm{ss}} \mathbf{E} \mathbf{s}_{\mathrm{s}}+\mathbf{H}_{\mathrm{ms}} \mathbf{s}_{\mathrm{m}}+\left(\mathbf{I}_{K} \otimes \mathbf{F}\right) \mathbf{n}_{\mathrm{s}},
\end{aligned}
$$

where $\mathbf{E}$ is the MU-VFDM precoder, constructed by the SAPs to cancel the interference from the SCs to the MC as detailed further ahead. In (1), $\mathbf{n}_{\mathrm{m}} \in \mathcal{N C}\left(0, \sigma_{n}^{2} \mathbf{I}_{N}\right)$ and $\mathbf{n}_{\mathrm{s}} \in \mathcal{N C}\left(0, \sigma_{n}^{2} \mathbf{I}_{K N}\right)$ are the thermal noise vectors.

The equivalent channel matrix for the $\mathrm{MC}, \mathbf{H}_{\mathrm{mm}} \in \mathcal{C}^{N \times N}$, is obtained by summing up all the contributions of the MUEs. Consequently, $\mathbf{H}_{\mathrm{mm}}=\sum_{j=1}^{M} \mathbf{B}_{j} \mathbf{F} \mathcal{T}\left(\mathbf{h}_{\mathrm{mm}}^{(1, j)}\right) \mathbf{A} \mathbf{F}^{-1}$, where, $\mathbf{F}^{-1}$ is the Inverse DFT (IDFT) matrix at the MBS, $\mathbf{A}$ is a $(N+L) \times N$ cyclic prefix insertion matrix and $\mathcal{T}\left(\mathbf{h}_{\mathrm{mm}}^{(\cdot, \cdot)}\right) \in$ $\mathcal{C}^{N \times(N+L)}$ is a Toeplitz matrix constructed from the $\mathbf{h}_{\text {mm }}^{(\cdot, \cdot)}$ channel coefficients as done in [8].

Now, let $\mathbf{H}_{\mathrm{ss}}^{(i, j)}=\mathcal{T}\left(\mathbf{h}_{\mathrm{ss}}^{(i, j)}\right)$ be the $N \times(N+L)$ matrix representing the link from the SAP $i$ to the SUE $j$. By defining

$$
\mathbf{H}_{\mathrm{ss}}^{\prime}=\left[\begin{array}{ccc}
\mathbf{H}_{\mathrm{s}}^{(1,1)} & \cdots & \mathbf{H}_{\mathrm{ss}}^{(1, K)} \\
\mathbf{H}_{\mathrm{ss}}^{(2,1)} & \cdots & \mathbf{H}_{\mathrm{ss}}^{(2, K)} \\
\vdots & \ddots & \vdots \\
\mathbf{H}_{\mathrm{ss}}^{(K, 1)} & \cdots & \mathbf{H}_{\mathrm{ss}}^{(K, K)}
\end{array}\right],
$$

we can write the overall equivalent SAPs to SUEs channel, as $\mathbf{H}_{\mathrm{ss}}=\left(\mathbf{I}_{K} \otimes \mathbf{F}\right) \mathbf{H}_{\mathrm{ss}}^{\prime} \in \mathcal{C}^{K N \times K(N+L)}$. We follow a similar approach for the interfering link from the MBS to the SUEs. Then, $\mathbf{H}_{\mathrm{ms}}^{\prime}=\left[\mathbf{H}_{\mathrm{ms}}^{(1,1)}, \mathbf{H}_{\mathrm{ms}}^{(1,2)}, \ldots, \mathbf{H}_{\mathrm{ms}}^{(1, K)}\right]^{\mathrm{T}}$, where $\mathbf{H}_{\mathrm{ms}}^{(1, j)}=\mathcal{T}\left(\mathbf{h}_{\mathrm{ms}}^{(1, j)}\right) \mathbf{A F}^{-1} \in \mathcal{C}^{N \times N}$ is the interfering link from the MBS to the SUE $j$. As before, the overall equivalent channel is $\mathbf{H}_{\mathrm{ms}}=\left(\mathbf{I}_{K} \otimes \mathbf{F}\right) \mathbf{H}_{\mathrm{ms}}^{\prime} \in \mathcal{C}^{K N \times N}$. To represent the overall interfering link from the SAPs to the MUEs, $\mathbf{H}_{\mathrm{sm}} \in \mathcal{C}^{N \times K(N+L)}$, we proceed as done above for $\mathbf{H}_{\mathrm{mm}}$. First, the equivalent channel from each of the SAP to the MUEs is constructed, in the form $\mathbf{H}_{\mathrm{sm}}^{(i, \cdot)}=\sum_{j=1}^{M} \mathbf{B}_{j} \mathbf{F} \mathcal{T}\left(\mathbf{h}_{\mathrm{sm}}^{(i, j)}\right)$. Then, the overall interfering link matrix from the SAPs to the MUEs is obtained by aggregating $\mathbf{H}_{\mathrm{sm}}^{(i, \cdot)}$, where $i \in[1, K]$, as $\mathbf{H}_{\mathrm{sm}}=\left[\mathbf{H}_{\mathrm{sm}}^{(1, \cdot)}, \ldots, \mathbf{H}_{\mathrm{sm}}^{(K, \cdot)}\right]$. To cancel the interference towards the MC, adhering to the overlay cognitive paradigm, the MU-VFDM precoder $\mathbf{E}$ must satisfy the interference cancelation constraint given by

$$
\mathbf{H}_{\mathrm{sm}} \mathbf{E}=\mathbf{0},
$$

where $\mathbf{E}$ is defined as

$$
\mathbf{E}=\bigoplus_{i=1}^{K} \mathbf{E}_{i}
$$

In (4), $\bigoplus$ is the direct sum operator [11] applied to the $K$ precoders used by the SAPs. Note that, for the condition (3) to be fulfilled, $\mathbf{E}$ must span the null space of $\mathbf{H}_{\mathrm{sm}}$. At this stage, we assume that no cooperation between the SAPs is 
required to design $\mathbf{E}$. Then, (3) is satisfied if the following holds

$$
\mathbf{H}_{\mathrm{sm}}^{(i, \cdot)} \mathbf{E}_{i}=\mathbf{0} \quad \forall i \in[1, K] .
$$

Therefore, we can focus on a single SAP $i$ at a time, designing a precoder $\mathbf{E}_{i}$ able to satisfy (5), and thus, (3). This allows the precoder computation to be carried out disjointly by the SAPs, resulting in a simpler architecture and lower signaling through the backhaul. Let $\mathbf{H}_{\mathrm{sm}}^{(i, \cdot)}=\mathbf{L Q}$ be its LQ decomposition. By definition, $\mathbf{L} \in \mathcal{C}^{N \times(N+L)}$ is a lower triangular matrix and $\mathbf{Q} \in \mathcal{C}^{(N+L) \times(N+L)}$ is an orthogonal matrix. By construction, $\operatorname{rank}(\mathbf{H}) \leq N$, thus we can find at least the last $L$ orthonormal columns of $\mathbf{Q}^{\mathrm{H}}$ lying onto the kernel of $\mathbf{H}_{\mathrm{sm}}^{(i, \cdot)}$. Then, by defining

$$
\mathbf{E}_{i} \triangleq\left[\mathbf{q}_{N+1}^{\mathrm{H}}|\cdots| \mathbf{q}_{(N+L)-1}^{\mathrm{H}} \mid \mathbf{q}_{N+L}^{\mathrm{H}}\right],
$$

we constuct a precoder that fulfills (5). We remark that a perfect knowledge of $\mathbf{H}_{\mathrm{sm}}^{(i, \cdot)}$ is needed to achieve the nulling condition in (3). In Secs. IV and V we discuss the effect of imperfect CSI on the performance of both the MC and SC systems.

Considering the structure of $\mathbf{E}_{i}$, note that each SAP $i$ faces a dimensionality constraint. Consequently, by aggregating the $K$ individual zero mean, unit norm symbol vectors $\mathbf{s}_{\mathrm{s}}^{(i)}$, of dimension $L$, the overall $\mathrm{SC}$ transmit vector $\mathbf{s}_{\mathrm{s}}$ has dimension $K L$.

Such a precoder projects the signal over the null-space of the interfering link as done in Vandermonde-subsapce Frequency Division Multiplexing (VFDM) [12]. The multi-user SC system exploits the left-over resources by the $\mathrm{MC}$, represented by the redundancy introduced by the MBS to combat inter block interference (e.g., cyclic prefix), to transmit without generating interference to the legacy system. The reader is advised to refer to [12], [8], and references therein, for more details.

\section{MU-VFDM PRECODER DESIGN}

The MU-VFDM precoder assures a harmless coexistence between the SCs and the MC. Nevertheless, the precoder $\mathbf{E}$ does not deal with the multi-user interference generated in the SCs. In [9], by exploiting the full coordination among the SAPs, we proposed a practical transmit scheme to deal with the multi-user interference at the SC system. For clarity, let

$$
\widetilde{\mathbf{H}}_{\mathrm{ss}}=\mathbf{H}_{\mathrm{ss}} \mathbf{E},
$$

of dimension $K N \times K L$. To overcome the dimensionality issue, and to be able to perform a regularized inverse beamforming (RIBF) [13], we introduced the load rate, i.e., the ratio between the number of dimensions at the transmitter and the ones at the receiver as

$$
\beta=\frac{\psi_{t x}}{\psi_{r x}} .
$$

RIBF is feasible when $\beta \geq 1$. Let $\psi_{t x}=\gamma_{t x} L$ and $\psi_{r x}=$ $\gamma_{r x} N$, where $\gamma_{t x}, \gamma_{r x} \in \mathbb{N}^{\star}$ are parameters related respectively to the transmitter and receiver (e.g. SC density, number of antenna at the receiver/transmitter), while $N$ and $L$ are fixed due to the OFDMA symbol structure. Let $\mathbf{u}_{\mathrm{s}} \in \mathcal{C}^{\gamma_{r x} K N \times 1}$ be a new aggregate $\mathrm{SC}$ transmit vector, such that

$$
\mathbf{s}_{\mathrm{s}}=\boldsymbol{\Phi} \mathbf{u}_{\mathrm{s}}
$$

where $\boldsymbol{\Phi} \in \mathcal{C}^{\gamma_{t x} K L \times K N}$ is the RIBF precoder, defined as

$$
\boldsymbol{\Phi}=\overline{\mathbf{H}}_{\mathrm{ss}}^{\mathrm{H}}\left(\frac{\sigma_{n}^{2}}{P_{s}} \mathbf{I}_{K N}+\overline{\mathbf{H}}_{\mathrm{ss}} \overline{\mathbf{H}}_{\mathrm{ss}}^{\mathrm{H}}\right),
$$

and normalized by $\sqrt{E_{\boldsymbol{\Phi}}}=\sqrt{\operatorname{tr}\left(\boldsymbol{\Phi} \boldsymbol{\Phi}^{\mathrm{H}}\right)}$, s.t. $\operatorname{tr}\left(\boldsymbol{\Phi} \boldsymbol{\Phi}^{\mathrm{H}}\right)=1$. The signal model (1) now reads

$$
\begin{aligned}
\mathbf{y}_{\mathrm{m}} & =\mathbf{H}_{\mathrm{mm}} \mathbf{s}_{\mathrm{m}}+\mathbf{F} \mathbf{n}_{\mathrm{m}} \\
\mathbf{y}_{\mathrm{s}} & =\mathbf{H}_{\mathrm{ss}} \mathbf{W} \mathbf{u}_{\mathrm{s}}+\mathbf{H}_{\mathrm{ms}} \mathbf{x}_{\mathrm{m}}+\nu_{\mathrm{s}},
\end{aligned}
$$

where $\mathbf{W}=\mathbf{E} \boldsymbol{\Phi} \in \mathcal{C}^{\gamma_{t x} K(N+L) \times K N}$ is the overall precoder. Now, $\mathbf{s}_{\mathrm{s}}$ has dimension $\gamma_{t x} K L, \mathbf{E} \in \mathcal{C}^{\gamma_{t x} K(N+L) \times \gamma_{t x} K L}$, which yields $\widetilde{\mathbf{H}}_{\mathrm{ss}} \in \mathcal{C}^{\gamma_{r x} K N \times \gamma_{t x} K L}, \mathbf{H}_{\mathrm{sm}} \in \mathcal{C}^{N \times \gamma_{t x} K(N+L)}$ and $\mathbf{y}_{\mathrm{s}} \in \mathcal{C}^{\gamma r x K N \times 1}$.

\section{CHANNEL ESTIMATION PROTOCOL}

One of the main issues related to a MU-VFDM practical implementation is the robustness against imperfect CSIT. A study of its performance under this assumption has to be performed to validate the proposed technique. In this section, we aim to take a step further in this direction by devising a practical channel estimation protocol for the SAPs, to be used in a realistic MU-VFDM implementation. The infinite backhaul capacity assumption made in Sec. II allows us to isolate the effect of a bad channel estimation onto the performance of the two-tiered network. The impact of the quantization of the CSI when the network operates under limited backhaul capacity will be the subject of future research. Note that, at the primary system, the LTE specifications mandate performance requirements for the transmission, but let complete freedom in channel estimation implementation [14]. Moreover, in [15] and reference therein, it is shown that, to cope with the aforementioned requirements in a practical scenario, the MUEs will likely need to adopt a precoder based transmit strategy, thus one of the possibilities is that an uplink channel estimation be performed. Therefore, even though different from a standard pilot-based approach, we assume that a 2steps channel estimation procedure based on preamble-training symbols [14] is performed in the MC, as depicted in Fig. 2: a) Uplink (UL) channel estimation, where the MUEs send OFDM training symbols, and the MBS estimates the channel; b) Downlink (DL) channel estimation, where the MBS sends in the downlink OFDM training symbols, and the MUEs estimate the channel. Considering a block fading channel of coherence time $T$, we suppose that channel estimation in the $\mathrm{MC}$ is performed during $\tau \leq T$. Therefore, $T-\tau$ is the available time for transmission, before new channel estimations are required. In the following, we will focus on the SC system channel estimation protocol. For simplicity, we divide $\tau$ into two phases, $\tau_{1}$ and $\tau_{2}$ : the UL channel estimation phase, during $\tau_{1}$, and the DL channel estimation phase, during $\tau_{2}$. A graphical representation of the estimation and transmission times is provided in Fig. 3. 


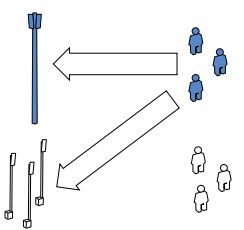

(a) UL

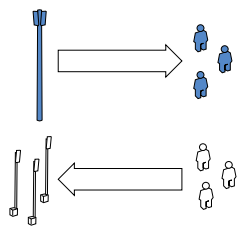

(b) DL
Figure 2. Channel estimation phases.

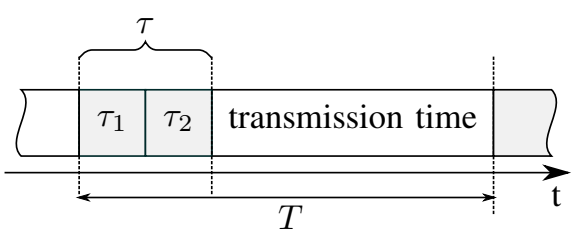

Figure 3. Channel estimation and transmission times.

\section{A. UL channel estimation $\left(\tau_{1}\right)$}

During $\tau_{1}$, as depicted in Fig. 2(a), the MUEs transmit OFDM training symbols used by the MBS to estimate the channels and perform downlink resource block allocation and input power optimization. Note that, each transmitted training symbol is received by both the MBS and the SAPs. We assume that, in this phase, the transmission is slotted in time, thus $\tau_{1}$ is divided into $M$ slots. Therefore, each MUE transmits during a time slot of length $\tau_{1} / M$. Consider the SC system, in the following we show how the CSIT w.r.t. the interfering link towards the MUEs can be acquired. Since the interference cancelation precoder is computed disjointly by the SAPs, then we can focus on a single SAP to describe the procedure. The received signal at $\mathrm{SAP} i$ from $\mathrm{MUE} j$ is

$$
\mathbf{y}_{\mathrm{s}}^{(j, i)}(t)=\mathbf{H}_{\mathrm{ms}}^{(j, i)} \mathbf{x}_{\mathrm{m}}+\nu_{\mathrm{s}}^{(i)}(t),
$$

where $\mathbf{x}_{\mathrm{m}}=\frac{1}{\sqrt{N}}[1, \ldots, 1]$ is a unit norm training symbol vector, $\mathbf{H}_{\mathrm{ms}}^{(j, i)}=\mathbf{F} \mathcal{T}\left(\mathbf{h}_{\mathrm{ms}}^{(j, i)}\right) \mathbf{A} \mathbf{F}^{-1}$ is the overall diagonal matrix and $t$ is time. Due to channel reciprocity, $\mathbf{H}_{\mathrm{ms}}^{(j, i)}=\mathbf{H}_{\mathrm{sm}}^{(i, j)}$, and the channel estimation $\widehat{\mathbf{H}}_{\mathrm{sm}}^{(i, j)}$ is given by

$$
\begin{aligned}
{\left[\widehat{\mathbf{H}}_{\mathrm{sm}}^{(i, j)}\right]_{n, n} } & =\frac{M \sqrt{N}}{\tau_{1}} \sum_{t=1}^{\frac{\tau_{1}}{M}}\left[\mathbf{y}_{\mathrm{s}}^{(j, i)}(t)\right]_{n} \\
& =\left[\mathbf{H}_{\mathrm{sm}}^{(i, j)}\right]_{n, n}+\frac{M \sqrt{N}}{\tau_{1}} \sum_{t=1}^{\frac{\tau_{1}}{M}}\left[\nu_{\mathrm{s}}^{(i)}(t)\right]_{n} .
\end{aligned}
$$

Let $\widehat{\mathbf{g}}=\mathbf{F}^{-1}\left[\left[\widehat{\mathbf{H}}_{\mathrm{sm}}^{(i, j)}\right]_{1,1}, \ldots,\left[\widehat{\mathbf{H}}_{\mathrm{sm}}^{(i, j)}\right]_{N, N}\right]^{\mathrm{T}}$ be the time domain version of $\widehat{\mathbf{H}}_{\mathrm{sm}}^{(i, j)}$. Then, by taking the first $L+1$ components of $\widehat{\mathbf{g}}$, each corresponding to the number of paths in the considered channel model, we define

$$
\widehat{\mathbf{h}}_{\mathrm{sm}}^{(i, j)} \triangleq\left[\widehat{\mathbf{g}}_{1}, \ldots, \widehat{\mathbf{g}}_{L+1}\right]^{\mathrm{T}} .
$$

The $i^{t h}$ SAP can finally construct $\mathcal{T}\left(\widehat{\mathbf{h}}_{\mathrm{sm}}^{(i, j)}\right)$ during the $j^{t h}$ time slot in $\tau_{1}$. We assume that, at this point, each SAP is aware of the resource block allocation performed by the MBS. Using this information, the $i^{\text {th }} \mathrm{SAP}$ can construct $\mathbf{B}_{j}$. Then,
$\mathbf{H}_{\mathrm{sm}}^{(i, j)}=\mathbf{B}_{j} \mathbf{F} \mathcal{T}\left(\widehat{\mathbf{h}}_{\mathrm{sm}}^{(i, j)}\right)$ is computed and the precoder $\mathbf{E}_{i}$ is derived, according to the procedure described in Sec. II.

\section{B. DL channel estimation $\left(\tau_{2}\right)$}

During $\tau_{2}$, as depicted in Fig. 2(b), training symbols are transmitted simultaneously in both MC and SCs. From the point of view of the MC, the MBS transmits to the MUEs a unit norm Walsh-Hadamard (W-H) sequence [16] $\mathbf{x}_{\mathrm{m}}$ of length $\tau_{2}$ at each subcarrier. Note that, $\tau_{2}$ size depends on the number of both subcarriers and performed channel estimations. This is done to allow $\mathbf{H}_{\mathrm{mm}}^{(1, j)}$ to be known at the $j^{\text {th }} \mathrm{MUE}$, for the equalization of the received signal during data transmission. The structure of the received signal is the same for each MUE, hence we focus on $j^{t h}$ MUE and write

$$
\mathbf{y}_{\mathrm{m}}^{(1, j)}(t)=\mathbf{H}_{\mathrm{mm}}^{(1, j)} \mathbf{X}_{\mathrm{m}}+\Upsilon_{\mathrm{m}}^{(j)}(t)
$$

where

$$
\begin{aligned}
\mathbf{X}_{\mathrm{m}} & =\left[\mathbf{x}_{\mathrm{m}}|\cdots| \mathbf{x}_{\mathrm{m}}\right]^{\mathrm{T}} \text { and } \\
\Upsilon_{\mathrm{m}}^{(j)}(t) & =\mathbf{F}\left[\boldsymbol{\nu}_{\mathrm{m}}^{(j)}(1)|\cdots| \boldsymbol{\nu}_{\mathrm{m}}^{(j)}\left(\tau_{2}\right)\right]
\end{aligned}
$$

are of size $\left(N \times \tau_{2}\right)$. At the end of $\tau_{2}$, the $j^{\text {th }}$ MUE estimates the channel to the MBS as

$$
\begin{aligned}
{\left[\widehat{\mathbf{H}}_{\mathrm{mm}}^{(1, j)}\right]_{n, n} } & =\left[\mathbf{y}_{\mathrm{m}}^{(1, j)}(t) \mathbf{X}_{\mathrm{m}}^{\mathrm{T}}\right]_{n, n} \\
& =\left[\mathbf{H}_{\mathrm{mm}}^{(1, j)}\right]_{n, n}+\left[\Upsilon_{\mathrm{m}}^{(j)}(t) \mathbf{X}_{\mathrm{m}}^{\mathrm{T}}\right]_{n, n} .
\end{aligned}
$$

Note that (15) is only possible due to the property of W-H sequences $\mathbf{X}_{\mathrm{m}} \mathbf{X}_{\mathrm{m}}^{\mathrm{T}}=\mathbf{I}$. Then, we proceed to find $\widehat{\mathbf{h}}_{\mathrm{mm}}^{(1, j)}$ as done for $\widehat{\mathbf{h}}_{\mathrm{sm}}^{(1, j)}$ in Sec. IV-A.

Turning our focus back to the SC system, we recall that its cognitive capabilities and the similarity between an MUE and an SUE allow us to assume that the SUEs know the physical layer characteristics of the MC. It is clear from (10) that, to design the $\boldsymbol{\Phi}$ precoder, the SAPs need to know the CSI with respect to each SUEs. As depicted in Fig. 2(b) each SUE transmits training symbols to the SAPs for channel estimation purposes. For simplicity, we assume that all SUEs transmit in a synchronized fashion. Techniques to allow this synchronized transmission or the effects of asynchronous transmission are out of the scope of this work and will be dealt in the future. To avoid interference towards the MUEs, the SUEs make use of the structure of the pilot symbols used in the channel estimation process into the MC. By definition of $\mathbf{x}_{\mathrm{m}}, \tau_{2}-1$ orthonormal W-H sequences can be found. Therefore, for $K \leq\left(\tau_{2}-1\right)$, up to $\tau_{2}-1$ SUEs can select a unique $\mathrm{W}$ $\mathrm{H}$ sequence $\mathbf{x}_{\mathrm{s}}^{(i)}$ as its training symbols, such that

$$
\mathbf{x}_{\mathrm{s}}^{(i) \mathrm{T}} \mathbf{x}_{\mathrm{m}}=0, \forall i \in[1, K] .
$$

We note that, this approach is scalable only up to $K \sim N$ SAPs/SUEs. On the other hand, if we consider the parameters of a realistic operative scenario (i.e., $N \sim \alpha * 10^{\{2,3\}}, \alpha \in \mathbb{Z}_{+}$), we can easily see that a scalability issue would arise only in practical network deployment involving an unlikely number of SAPs. Moreover, at this stage, we do not impose any 
mechanism to chose the $\mathrm{W}-\mathrm{H}$ sequences in the SC network. Depending on the designer's goal, one of the many schemes proposed in the literature, i.e. [17], [18], could be adopted.

The received signal during $\tau_{2}$ at the $j^{\text {th }}$ MUE is

$$
\begin{aligned}
\mathbf{y}_{\mathrm{m}}^{(1, j)}(t)= & \mathbf{H}_{\mathrm{mm}}^{(1, j)} \mathbf{X}_{\mathrm{m}}+ \\
& \sum_{i=1}^{K} \mathbf{B}_{j} \mathbf{F} \mathcal{T}\left(\mathbf{h}_{\mathrm{sm}}^{(i, j)}\right) \mathbf{F}^{-1} \mathbf{X}_{\mathrm{s}}^{(j)}+\Upsilon_{\mathrm{m}}^{(j)}(t),
\end{aligned}
$$

where $\Upsilon_{\mathrm{m}}^{(j)}(t)=\mathbf{B}_{j} \mathbf{F}\left[\boldsymbol{\nu}_{\mathrm{m}}^{(j)}(1)|\cdots| \boldsymbol{\nu}_{\mathrm{m}}^{(j)}\left(\tau_{2}\right)\right]$, and the estimate of the channel is given as in (15), since $\sum_{i=1}^{K} \mathbf{B}_{j} \mathbf{F} \mathcal{T}\left(\mathbf{h}_{\mathrm{sm}}^{(i, j)}\right) \mathbf{F}^{-1} \mathbf{X}_{\mathrm{s}}^{(j)} \mathbf{X}_{\mathrm{m}}^{\mathrm{T}}=\mathbf{0}$. Hence, no interference is generated from the SUEs towards the MUEs.

The received signal from the SUEs at the $j^{\text {th }} \mathrm{SAP}$ is

$$
\begin{aligned}
\mathbf{y}_{\mathrm{s}}^{(\cdot, j)}(t)= & \sum_{i=1}^{K} \mathbf{H}_{\mathrm{ss}}^{(i, j)} \mathbf{X}_{\mathrm{s}}^{(i)}+ \\
& \mathbf{F T}\left(\mathbf{h}_{\mathrm{ms}}^{(1, j)}\right) \mathbf{F}^{-1} \mathbf{X}_{\mathrm{m}}+\Upsilon_{\mathrm{s}}^{(j)}(t),
\end{aligned}
$$

and, since the $j^{t h}$ SAP needs an estimate of the channels towards each SUE, then it performs $K$ different channel estimations to find $\mathbf{H}_{\mathrm{ss}}^{(i, j)}, \forall i \in[1, K]$. We focus on the link connecting the $j^{\text {th }} \mathrm{SAP}$ and $i^{\text {th }} \mathrm{SUE}$, the remaining channels are estimated similarly. Due to the previous consideration in the SC system, we assume that the $j^{t h}$ SAP knows $\mathbf{X}_{\mathrm{s}}^{(i)}, \forall i \in$ $[1, K]$. Therefore, $\widehat{\mathbf{H}}_{\mathrm{ss}}^{(i, j)}$ is given by

$$
\begin{aligned}
{\left[\widehat{\mathbf{H}}_{\mathrm{ss}}^{(i, j)}\right]_{n, n,} } & =\left[\mathbf{y}_{\mathrm{s}}^{(\cdot, i)}(t) \mathbf{X}_{\mathrm{s}}^{(i) \mathrm{T}}\right]_{n, n} \\
& =\left[\mathbf{H}_{\mathrm{ss}}^{(i, j)}\right]_{n, n}+\left[\Upsilon_{\mathrm{s}}^{(j)}(t) \mathbf{X}_{\mathrm{s}}^{(i) \mathrm{T}}\right]_{n, n},
\end{aligned}
$$

since $\mathbf{F} \mathcal{T}\left(\mathbf{h}_{\mathrm{ms}}^{(1, j)}\right) \mathbf{F}^{-1} \mathbf{X}_{\mathrm{m}}^{(j)} \mathbf{X}_{\mathrm{s}}^{(i) \mathrm{T}}=\mathbf{0}$. Again, due to the use of the $\mathrm{W}-\mathrm{H}$ training symbols, no interference is generated from the MBS towards the $j^{\text {th }}$ SAP. We proceed to find $\widehat{\mathbf{h}}_{\mathrm{ss}}^{(i, j)}$ as done for in Sec. IV-A. Finally, at the end of $\tau_{2}$, the precoder W can be constructed, as described in Sec. III. After the channel estimation phase $\tau$, both systems can engage in the transmission phase during $T-\tau$.

\section{NuMERICAL RESULTS}

In this section, we illustrate the impact of the channel estimation protocol on the performance of MU-VFDM. In the Monte Carlo simulations, we consider an OFDMA MC system downlink with $M=4$ MUEs, characterized by $N=32$ active subcarriers, cyclic prefix of length $L=8$. The ratio $\frac{L}{N}=\frac{1}{4}$ is peculiar to the extended mode of a typical LTE configuration [19], and the values of $N, L$ have been chosen merely to provide feasible computational time. In the SC system we consider $K=3$ SAPs/SUEs with a load rate $\beta=1$. Noise and channel vectors are generated as described in Sec. II.

Let

$$
\begin{aligned}
C_{\mathrm{m}}^{\mathrm{SUM}, \mathrm{P}} & =\frac{1}{N+L} \sum_{i=1}^{N} \log _{2}\left(1+\rho_{i}\right) \text { and } \\
C_{\mathrm{s}}^{\mathrm{SUM}, \mathrm{P}} & =\frac{1}{N+L} \sum_{i=1}^{\gamma_{r x} K N} \log _{2}\left(1+\rho_{i}\right)
\end{aligned}
$$

be the sum-rate for the MC and SCs respectively, when perfect CSIT is available, where $\rho$ denotes the Signal to Interference plus Noise Ratio (SINR). As a result of the relaxation of the perfect CSIT assumption at the SAPs, the interference cancelation constraint in (3) is not fulfilled anymore and the MUEs suffer interference from the SAPs' transmission, translated into a sum-rate loss. For the SC system, the precoder $\mathbf{W}$ is based on an imperfect channel estimation affecting the achievable sum-rate of the SC system, because of multi-user interference. From [20], we know that, given the SINR for the perfect CSIT, we can define an effective SINR value as

$$
\rho_{\mathrm{eff}}=\frac{\rho^{2} \tau}{1+(1+\tau) \rho},
$$

where we assume equal transmit power for training and data symbols. Therefore, let

$$
\begin{aligned}
C_{\mathrm{m}}^{\mathrm{SUM}, \mathrm{I}} & =\frac{T-\tau}{T(N+L)} \sum_{j=1}^{N} \log _{2}\left(1+\rho_{\mathrm{eff}, j}\right) \text { and } \\
C_{\mathrm{s}}^{\mathrm{SUM}, \mathrm{I}} & =\frac{T-\tau}{T(N+L)} \sum_{j=1}^{K N} \log _{2}\left(1+\rho_{\mathrm{eff}, j}\right)
\end{aligned}
$$

be the sum-rate of the MC and the SCs respectively, when the proposed channel estimation protocol is adopted. Note that, at this stage, we do not consider any interference from the MBS to the SUEs, in order to be able to evaluate the effect of the imperfect channel estimation at the SAPs.

In Fig. 4, the ratio between the rate obtained with imperfect CSIT and the rate obtained with perfect CSIT, is computed for the $\mathrm{MC}$ and the $\mathrm{SC}$ system, as different $\tau / T$ proportions are chosen for $\mathrm{SNR} \in\{0,10,20\} \mathrm{dB}$. The optimal $\tau$ is dependent on the SNR in both cases. For the MC, we notice that, for medium and high SNR values, the sum-rate scales nearly linear with the pre-log factor, hence the best performance is achieved for the minimum value given the considered parameters, $\tau=0.08 T$. In general the performance for different SNR values is very similar. On the other hand, for the SC system, a bigger training time results always in a power gain, thus the optimal $\tau$ is always different. Moreover, by comparing the two systems we can see that a worse CSIT affects mainly the sum-rate of the SC system, penalized especially at very low SNR. In fact, the RIBF precoder adopted in MU-VFDM is very sensitive to channel estimation errors, hence the SC system performance is strongly dependent on the SNR values. In Fig. 5 the SNR is kept constant at $15 \mathrm{~dB}$, while the load rate $\beta \in\{1,1.5,2\}$. Conversely, in Fig. 6, three subcarriers values are tested, $N \in\{24,32,40\}$. In both cases the technique shows almost constant performance for the $\mathrm{MC}$, where the optimal $\tau$ corresponds to the minimum value. The SCs rate-loss is more evident and, in general, the bigger $N$ the worse the performance. Nevertheless, the simulation shows a clear improvement as the network becomes more dense (i.e., bigger $\beta$ ), thus, in order to guarantee a good sum-rate to the SCs, a trade-off between $N$ and $\beta$ is likely to be found. 

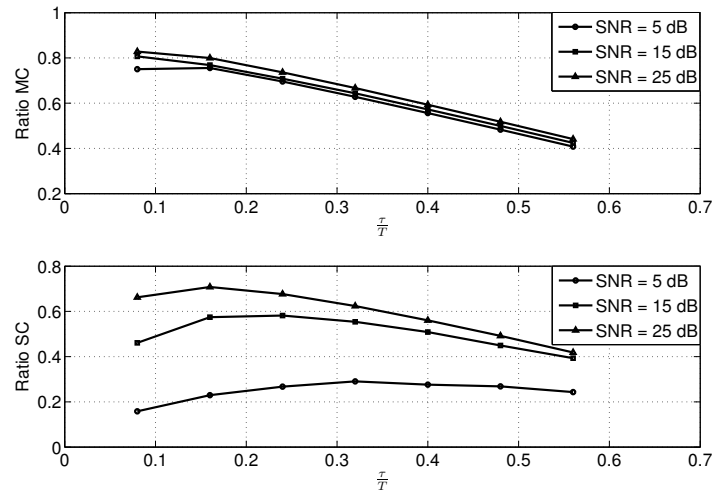

Figure 4. Ratio between the rate obtained with imperfect CSIT and the rate obtained with perfect CSIT for MC and SC system as SNR changes.
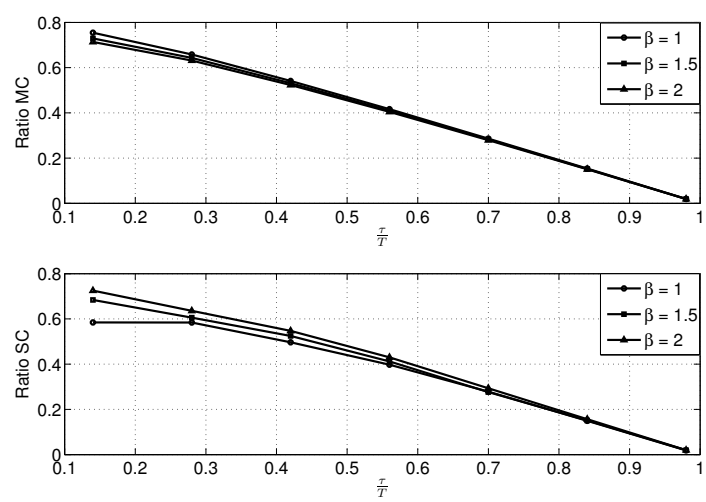

Figure 5. Ratio between the rate obtained with imperfect CSIT and the rate obtained with perfect CSIT for MC and SC system as $\beta$ changes.
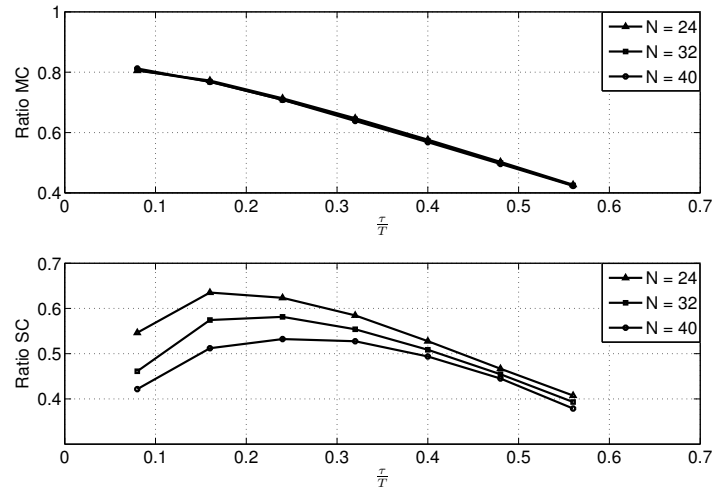

Figure 6. Ratio between the rate obtained with imperfect CSIT and the rate obtained with perfect CSIT for MC and SC system as $N$ changes.

\section{CONCLUSION}

In this work, we have revisited a dynamic spectrum access overlay technique called MU-VFDM. This technique allows the deployment of SCs inside of an MC, while generating no interference to the latter at the cost of perfect CSIT at the SAPs. In the current work, we have moved one step closer to a realistic setting, by relaxing the perfect CSIT assumption. With that in mind, we have devised a suitable channel estimation protocol, taking into consideration the requirements of MUVFDM's cascaded precoder structure. As expected, analysis of the sum-rate shows that imperfect CSI incurs a loss at both systems. Then, the best compromise between training and data symbols has been discussed. The results presented herein reinforce our previous findings that MU-VFDM can be used to allow the coexistence of future SCs and MCs inside the same coverage area, sharing the same band.

The study of other implementation issues of MU-VFDM is an ongoing work. A finite backhaul capacity and clustered cooperation between the SAPs will be considered. Moreover, we plan to study how synchronization issues, critical aspect in a cognitive radio framework, can impact on the performance of both systems. All these results will allow the further implementation of a transmission testbed based on MU-VFDM to prove its effectiveness as a candidate for the future deployment of SCs.

\section{REFERENCES}

[1] H. Holma and A. Toskala. LTE for UMTS OFDMA and SC-FDMA Based Radio Access. 2009.

[2] J. Hoydis, M. Kobayashi, and M. Debbah. Green small-cell networks. IEEE Vehicular Technology Magazine, 6(1):37-43, 2011.

[3] Alcatel Lucent. Lightradio: Evolve your wireless broadband network for the new generation of applications and users. [Online] Available: http://www.alcatel-lucent.com/features/light_radio/index.html, 2010.

[4] A. Goldsmith, S.A. Jafar, I. Maric, and S. Srinivasa. Breaking spectrum gridlock with cognitive radios: An information theoretic perspective. Proceedings of the IEEE, 97(5):894-914, 2009.

[5] W. Zhang and U. Mitra. Spectrum shaping: A new perspective on cognitive radio (part I): Coexistence with coded legacy transmission. IEEE Transactions on Communications, 58(6):1857-1867, 2010.

[6] Y. Ma, D.I. Kim, and Z. Wu. Optimization of OFDMA-based cellular cognitive radio networks. IEEE Transactions on Communications, 58(8):2265-2276, 2010.

[7] S. Haykin. Cognitive radio: brain-empowered wireless communications. IEEE Journal on Selected Areas in Communications, 23(2):201-220, 2005.

[8] L.S. Cardoso, M. Maso, M. Kobayashi, and M. Debbah. Orthogonal LTE two-tier cellular networks. In IEEE International Conference on Communications, 2011.

[9] M. Maso, L. Cardoso, M. Debbah, and L. Vangelista. Orthogonal precoder for two-tiered networks. submitted for publication.

[10] D. Gesbert, M. Kountouris, R. W. Heath Jr., C.-B. Chae, and T. Salzer. Shifting the MIMO paradigm: From single user to multiuser communications. IEEE Signal Processing Magazine, 24(5):36-46, 2007.

[11] F. Jr. Ayres. Theory and Problems of Matrices. Mcgraw-Hill, 1967.

[12] L.S. Cardoso, M. Kobayashi, Ø. Ryan, and M. Debbah. Vandermonde frequency division multiplexing for cognitive radio. In Proceedings of the 9th IEEE Workshop on Signal Processing Advances in Wireless Communications, 2008.

[13] C.B. Peel, B.M. Hochwald, and A.L. Swindlehurst. A vectorperturbation technique for near-capacity multiantenna multiuser communication (part I): channel inversion and regularization. IEEE Transactions on Communications, 53(1):195-202, 2005.

[14] S. Sesia, I. Toufik, and M. Baker. LTE - The UMTS Long Term Evolution. 2009.

[15] D. Grieco, J-L. Pan, R. Olesen, and N. Shah. Uplink single-user MIMO for 3GPP LTE. In IEEE 18th International Symposium on Personal, Indoor and Mobile Radio Communications, 2007.

[16] H.F. Harmuth. Transmission of Information by Orthogonal Functions. Springer; 2nd ed., 1972.

[17] E. Alsusa and C.; Masouros. Adaptive code allocation for interference management on the downlink of DS-CDMA systems. IEEE Transactions on Wireless Communications, 7(7):2420 - 2424, 2008.

[18] Y. Zhou, J. Wang, and T.-S. Ng. Downlink transmission of broadband OFCDM systems - part V: Code assignment. IEEE Transactions on Wireless Communications, 7(11):4546-4557, 2008.

[19] 3GPP TR 25.814, Physical Layer Aspects for Evolved UTRA, v.2.0.0. $3 \mathrm{GPP}, 2006$.

[20] B. Hassibi and B. Hochwald. How much training is needed in multipleantenna wireless links? IEEE Transactions on Information Theory, 49(4):951-963, 2003. 